\title{
Analysis of the application of ideal gas equation of state
}

\author{
YarongWang $^{1 *}$ and PeirongWang ${ }^{2}$ \\ ${ }^{1}$ Baoding Electric Power VOC.\&TECH. College, Baoding, Hebei, 071051, China \\ ${ }^{2}$ Hebei Software Institute, Baoding, Hebei, 071000, China
}

\begin{abstract}
In nature, the molecules of real gas have a certain volume and have interaction force with each other. It is difficult to find the molecular motion law of real gas because of its complex properties. An ideal gas is an imaginary substance that does not exist in reality. Its molecules are elastic, non volume particles, and there is no interaction among them. This kind of gas is simple in nature and easy to be analyzed and calculated by simple mathematical relation. The introduction of the concept of ideal gas greatly simplifies the analysis of some thermodynamic problems.
\end{abstract}

\section{Introduction}

In thermal equipment, the energy transfer and conversion depend on the state change of working medium. The results of energy transfer and conversion are different due to different thermodynamic properties and state change processes of working medium. Therefore, in the study of conversion of heat and work, we must be familiar with the thermodynamic properties of common working medium. In nature, the molecules of real gas have volume, and there are some force interactions among molecules. When the specific volume of gas is not very large, these two factors have a certain impact on the molecular motion, which makes the molecular motion law complex. The volume of molecules and the interaction among molecules must be considered in engineering calculation. This makes the nature of the real gas more complex, the relationship of state parameters is more complex. In order to simplify the analysis and calculation in thermodynamics, the concept of ideal gas is put forward. It is considered that the molecules of ideal gas are elastic and non volume particles, and there are no any force interactions among molecules. In any equilibrium state, the expression describing the relationship among the basic state parameters of ideal gas is called the ideal gas equation of state.

\section{Ideal gas equation of state.}

\section{1 state equation}

The ideal gas follows Clapeyron equation. Clapeyron equation describes the relationship among the three state parameters of ideal gas in the same state, which is only applicable to ideal gas. It is also known as ideal gas equation of state. The equation has the following forms, where $p, v, T$ represents pressure, specific volume and temperature, respectively:

$$
\begin{aligned}
& p v=R T \\
& p V_{m}=R_{m} T \\
& p V=m R T \\
& p V=n R_{m} T
\end{aligned}
$$

Where

$\mathrm{V}_{\mathrm{m}}$ - molar specific volume, $\mathrm{m} 3 / \mathrm{kmol}$.

$\mathrm{n}$ - mole number, kmol.

$\mathrm{R}_{\mathrm{m}}$ - the universal gas constant, it is the same for all kinds of ideal gases, $\mathrm{J} /(\mathrm{kmol} \cdot \mathrm{K})$.

In the standard condition, the molar specific volume of all ideal gases is $22.4 \mathrm{~m}^{3} / \mathrm{kmol}$.

Therefore, the universal gas constant can be calculated by the following formula [1]

$$
\begin{aligned}
R_{m} & =\frac{p_{0} V_{m 0}}{T_{0}} \\
& =\frac{1.01325 \times 10^{5} \times 22.414}{273.15} \\
& =8314 \quad \mathrm{~J} /(\mathrm{kmol} \cdot \mathrm{K})
\end{aligned}
$$

$\mathrm{R}$ - gas constant, $\mathrm{J} /(\mathrm{kg} \bullet \mathrm{K})$.

It has nothing to do with the state of the gas, but it varies with the type of gas. That is to say, for the same gas, the value of gas constant is constant no matter in what state, but the constant value of different kinds of gas is different. 


$$
R_{m}=M \cdot R
$$

Where

$$
\mathrm{M} \text {-molar mass, } \mathrm{kg} / \mathrm{kmol} \text {. }
$$

Different gases have different molar masses. The thousand molar mass of a gas is numerically equal to its value of molecular weight.

The pressure in the equation is absolute pressure and the temperature is absolute pressure. The ideal gas equation of state is an approximate description of gas properties. When the gas density is large and the specific volume is very small, the relationship of $\mathrm{p}-\mathrm{v}-\mathrm{T}$ of real gas deviates from this equation significantly. Even at low density, the two are only roughly consistent. Only when the gas pressure is very low and the specific volume is very large, that is $\mathrm{p} \rightarrow 0, \mathrm{v} \rightarrow 0$, can the properties of the gas completely conform to the ideal gas equation of state [1]. Experiments have proved that when the pressure is low enough and the temperature is high enough, the specific volume of gas is large enough.

In practical calculation, many real gases can be treated as ideal gases with acceptable error. For example, the commonly used gases $\mathrm{O}_{2}, \mathrm{~N}_{2}, \mathrm{H}_{2}, \mathrm{CO}, \mathrm{CO}_{2}$ etc, as well as the air and flue gas composed of these gases, which are far away from the liquid area under normal pressure and temperature, can be treated as ideal gases. However, the steam generated in the boiler can not be treated as ideal gas because they are close to the liquid region. It must be specially pointed out that the water vapor contained in the flue gas and the atmosphere can still be studied as an ideal gas because of its small content. It can be seen that there is no obvious boundary between ideal gas and real gas. According to the allowable error range of engineering calculation, what kind of gas should be considered under what conditions should be determined [2].

\subsection{Application examples}

Examples: The pressure and temperature measured in the user pipe network of gas company to deliver natural gas to users are $200 \mathrm{MPa}$ and $275 \mathrm{~K}$ respectively. If the pipeline diameter is $50 \mathrm{~mm}$ and the natural gas flow rate is $5.5 \mathrm{~m} / \mathrm{s}$, please determine the mass flow rate and the volume flow rate under the standard state. The atmospheric pressure is $0.1 \mathrm{MPa}[3]$.

Solution

$$
\begin{aligned}
p & =p_{g}+p_{b} \\
& =200+0.1 \times 10^{6} \\
& =1.002 \times 10^{5} \quad \mathrm{~Pa}
\end{aligned}
$$

$T=275 \quad K$

$p_{0}=1.01325 \times 10^{5} \quad p_{a}$

$T_{0}=273 \quad K$

$$
\begin{aligned}
R & =\frac{R_{m}}{M} \\
& =\frac{8314}{16}=519.625 \quad \mathrm{~J} /(\mathrm{kg} \cdot \mathrm{K})
\end{aligned}
$$

For the gas constants, we can calculate the gas constant in the above way. In addition, we can also refer to the relevant literature to make the values of molecular weight and the gas constant more accurate. As shown in Table 1[4]. According to different accuracy requirements, we can choose different methods.

$$
\begin{aligned}
R & =\frac{R_{m}}{M} \\
& =\frac{8314}{16.043}=518.2 \quad \mathrm{~J} /(\mathrm{kg} \cdot \mathrm{K})
\end{aligned}
$$

Table 1. Gas constants of several ideal-gases

\begin{tabular}{c|c|c|c||c|c|c|c}
\hline Substance & $\begin{array}{c}\text { Molecular } \\
\text { formula }\end{array}$ & Molar mass & $\begin{array}{c}\mathbf{R} \\
{[\mathrm{J} /(\mathrm{kg} \cdot \mathrm{K})]}\end{array}$ & Substance & $\begin{array}{c}\text { Molecular } \\
\text { formula }\end{array}$ & Molar mass & $\begin{array}{c}\mathbf{R} \\
{[\mathrm{J} /(\mathrm{kg} \cdot \mathrm{K})]}\end{array}$ \\
\hline Hydrogen & $\mathrm{H}_{2}$ & 2.016 & 1124.0 & Nitrogen & $\mathrm{N} 2$ & 28.013 & 296.8 \\
\hline Helium & $\mathrm{He}$ & 4.003 & 2077.0 & Carbon monoxide & $\mathrm{CO}$ & 28.014 & 296.8 \\
\hline Methane & $\mathrm{CH}_{4}$ & 16.043 & 518.2 & Carbon dioxide & $\mathrm{CO}_{2}$ & 44.010 & 288.9 \\
\hline Ammonia & $\mathrm{NH}_{3}$ & 17.031 & 488.2 & Oxygen & $\mathrm{O}_{2}$ & 32.0 & 259.8 \\
\hline Water vapor & $\mathrm{H}_{2} \mathrm{O}$ & 18.015 & 461.5 & Air & & 28.97 & 287.0 \\
\hline
\end{tabular}




$$
\begin{aligned}
q_{V} & =\frac{\pi D^{2}}{4} c \\
& =\frac{\pi \times 0.05^{2}}{4} \times 5.5 \\
& =1.08 \times 10^{-2} \mathrm{~m}^{3} / \mathrm{s}
\end{aligned}
$$

According to the four different forms of the ideal gas equation of state, four methods are used to calculate the mass flow rate.

Method 1

According to equation (1)

$$
\begin{aligned}
v= & \frac{R T}{p} \\
= & \frac{519.625 \times 275}{1.002 \times 10^{5}} \\
= & 1.426 \quad \mathrm{~m}^{3} / \mathrm{kg} \\
q_{m} & =m=\frac{V}{v}=\frac{q_{V}}{v} \\
& =\frac{1.08 \times 10^{-2}}{1.426} \\
& =7.57 \times 10^{-3} \mathrm{~kg} / \mathrm{s}
\end{aligned}
$$

Method 2

According to equation (2)

$$
\begin{aligned}
v_{m} & =\frac{R_{m} T}{p} \\
& =\frac{8314 \times 275}{1.002 \times 10^{5}} \\
& =22.817 \mathrm{~m}^{3} / \mathrm{kmol} \\
v & =\frac{v_{m}}{M} \\
& =\frac{22.817}{16} \\
& =1.426 \mathrm{~m}^{3} / \mathrm{kg}
\end{aligned}
$$

The mass flow rate is the mass of the gas flowing through the unit time:

$$
\begin{aligned}
q_{m} & =m=\frac{V}{v}=\frac{q_{V}}{v} \\
& =\frac{1.08 \times 10^{-2}}{1.426} \\
& =7.57 \times 10^{-3} \mathrm{~kg} / \mathrm{s}
\end{aligned}
$$

Method 3

According to equation (3)

$$
\begin{aligned}
q_{m} & =m=\frac{p V}{R T} \\
& =\frac{1.002 \times 10^{5} \times 1.08 \times 10^{-2}}{519.625 \times 275} \\
& =7.57 \times 10^{-3} \mathrm{~kg} / \mathrm{s}
\end{aligned}
$$

Method 4

According to equation (4)

$$
\begin{aligned}
n= & \frac{p V}{R_{m} T} \\
= & \frac{1.002 \times 10^{5} \times 1.08 \times 10^{-2}}{8314 \times 275} \\
= & 4.733 \times 10^{-4} \mathrm{kmol} \\
q_{m} & =m=n M \\
& =4.733 \times 10^{-4} \times 16 \\
& =7.57 \times 10^{-3} \mathrm{~kg} / \mathrm{s}
\end{aligned}
$$

Although they are all not very complicated, it is obvious that the third form of equation, method 3 , is the simplest for this example.

The volume flow rate is calculated as follows

Method 1

According to equation (3)

$$
\begin{aligned}
V_{0} & =\frac{m R T_{0}}{p_{0}} \\
& =\frac{7.57 \times 10^{-3} \times 519.625 \times 273}{1.01325 \times 10^{5}} \\
& =1.06 \times 10^{-2} \mathrm{~m}^{3} / \mathrm{s}
\end{aligned}
$$

The volume flow rate is the volume of gas flowing through the unit time:

$$
\begin{aligned}
q_{V_{0}} & =V_{0} \\
& =1.06 \times 10^{-2} \quad \mathrm{~m}^{3} / \mathrm{s}
\end{aligned}
$$

Method 2

According to equation (3), under the condition of constant mass, there is

$$
m R=\frac{p V}{T}=\frac{p_{0} V_{0}}{T_{0}}
$$

Therefore

$$
\begin{aligned}
V_{0} & =\frac{p V}{T} \cdot \frac{T_{0}}{p_{0}} \\
& =\frac{1.002 \times 10^{5} \times 1.08 \times 10^{-2} \times 273}{275 \times 1.01325 \times 10^{5}} \\
& =1.06 \times 10^{-2} \mathrm{~m}^{3} / \mathrm{s}
\end{aligned}
$$




$$
\begin{aligned}
q_{V_{0}} & =V_{0} \\
& =1.06 \times 10^{-2} \quad \mathrm{~m}^{3} / \mathrm{s}
\end{aligned}
$$

\section{Conclusion}

The assumption of ideal gas can meet the requirements of thermal engineering design and calculation, and the complexity and workload of calculation are reduced, so it plays a special role in engineering thermodynamics. Therefore, ideal gas and ideal gas equation of state occupy a special position in engineering thermodynamics. The equation of state of ideal gas is an equation describing the relationship between the basic state parameters of ideal gas. There are several forms of the equation. In the use of ideal gas equation of state, we should pay attention to: absolute pressure must be used instead of gauge pressure; absolute temperature must be used instead of centigrade temperature; the units of each physical quantity must be coordinated. Several forms of the ideal gas equation of state are interlinked. When we use it, we just need to choose the simplest form. Through the analysis of examples, we can intuitively see the characteristics of different forms of equations in the application. For some vocational students who have just studied the basic courses, they often have no choice when facing different forms of equations. This analysis process is helpful for them to compare the use characteristics of different forms of equations, so as to better use the equations to solve more complex practical problems.

\section{References}

1. Zhu, M.S., Liu, Y., Lin, Z.Z., Peng, X.F. (2011) Engineering Thermodynamics. Tsinghua university press, Beijing.

2. Shang, Y.Q. (2007) Engineering Thermodynamics. China Electric Power Press, Beijing.

3. Tong, J.G., Wang, P.Y., Su, Y.K.(2008) Thermal engineering. Shanghai Jiaotong University Press.

4. Zhao, L, Engineering Thermodynamics (A Bilingual Textbook) (2012), China Building Industry Press, Beijing.

5. Jing, Z.H. (2009) Thermal Engineering, China Electric Power Press, Beijing.

6. Xu, Y.P., Ke, X.Y. (2012) Thermal Engineering. China Electric Power Press, Beijing.

7. He, Y.L. (2001) Essential analysis of engineering thermodynamics and solutions to typical problems, Xi'an Jiaotong University Press, Xi'an

8. Zhou, J.H., Liu, X. (2010) Specialized English for thermal power generation, China Electric Power Press, Beijing.

9. Yan, W.P. (2009) Specialized English for thermal Energy and Power Engineering (Third Edition), China Electric Power Press, Beijing.

10. Hu, Y.H. (2008) English in Thermal Energy and Power Engineering, Tianjin University Press, Tianjin .
11. Han, S.F., Ren, J.Y. (2014) Foundation and application of thermal engineering. Beijing University of Technology Press, Beijing.

12. Shen,W.D., Geng,T.J. (2007) Engineering Thermodynamics, Higher Education Press, Beijing.

13. Chen, G.T., Wang, Y.Z. (2007) Engineering Thermodynamics (Second Edition). Beijing University of Technology Press, Beijing.

14. Chen, Z.H. (2009) Basic Thermal Engineering, China Electric Power Press, Beijing.

15. Tang, L.P. (2013) Basic Thermal Engineering, China Electric Power Press, Beijing. 